Original Research Paper

\title{
Elliptic Weighted Problem with Indefinite Asymptotically Linear Nonlinearity
}

\author{
Hanadi Zahed and Laila A. Alnaser \\ Department of Mathematics, College of Science, Taibah University, Al-Madinah, Al-Munawarah, Saudi Arabia
}

\section{Article history}

Received: 18-10-2020

Revised: 06-02-2021

Accepted: 08-02-2021

Corresponding Author:

Hanadi Zahed

Department of Mathematics,

College of Science, Taibah

University, Al-Madinah, Al-

Munawarah, Saudi Arabia

Email: hanadi71@hotmail.com
Abstract: The objective of this paper is the study the following nonlinear elliptic problem involving a weight function:

$-\operatorname{div}(a(x) \nabla v)=f(x, u)$ in $\Omega$ and $u=0$ on $\partial \Omega$

where, $\Omega$ is a regular bounded subset $\mathbb{R}^{N}$ and $N \geq 2, a(x)$ is a nonnegative function and $f(x, t)$ is allowed to be sign-changing. We employ variational techniques to prove the existence of a nontrivial solution for the problem $(P)$, under some suitable assumptions, when the nonlinearity is asymptotically linear. Then, we prove by the same method the existence of positive solution when the function $f$ is superlinear and subcritical at infinity.

2010 Mathematics, Subject, classification: 35J05, 35J65, 35J20, 35J60, 35K57, 35J70.

Keywords: Elliptic Problem, Asymptotically Linear, Nonnegative Weight Function, Indefinite Nonlinearity, Variational Method

\section{Introduction with Main Results}

In the present paper, we investigate the existence result for the following nonlinear elliptic equation involving sign-changing nonlinearity:

$$
\left\{\begin{aligned}
-\operatorname{div}(a(x) \nabla u) & =f(x, u) & & \text { in } \Omega, \\
u & =0 & & \text { on } \partial \Omega,
\end{aligned}\right.
$$

where, $\Omega \subset \mathbb{R}^{N}, N \geq 2$, is a smooth bounded open set, $a(x)$ is a nonnegative function and also the function $f(x, t)$ is an indefinite nonlinearity.

This problem is nonlinear and in Physics, Dynamics and Biology nonlinear problems have many interest since they are able to explain the evolution of a system. If we change some parameters or the nonlinearity, the system undergoes transitions mainly the existence of solutions: We have the bifurcation phenomena. The problem (1.1) is the stationary position of the problem induced in 1952 by (Turing, 1952):

$$
\frac{\partial u}{\partial t}-\operatorname{div}(a(x) \nabla u)=f(x, u)
$$

which modules the interaction between species and chemicals in a morphogenesis phenomenon in Biology, where $u$ is the density and $f(x, t)$ represents the diffusion-interaction of substances. The term $-\operatorname{div}(a(x) \nabla u)$ indicatess the substance of diffusion through the given system.

When $a$ is a constant function, many authors have studied the problem (1.1) with asymptotically linear nonlinearity. For this reason, (Mironescu and Radulescu, 1993; 1996) considered the problem:

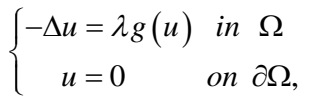

and supposed the assumptions:

(G1) $g: \mathbb{R} \rightarrow \mathbb{R}$ a $C^{1}$ positive function

(G2) The function $g$ is increasing

(G3) The function $g$ is convex

(G4) $\lim _{t \rightarrow+\infty} \frac{g(t)}{t}=\ell \in(0,+\infty)$.

When $f$ is superlinear and $\ell=(0,+\infty)$, the problem (1.2) was studied in (Brezis et al., 1996; Martel, 1997) and the references therein and it is generated to the $p$ Laplace operator in (Filippakis and Papageorgiou, 2006; Sanchón, 2007). The same problem with Bi-Laplace operator has been studied in (Arioli et al., 2005; Abid et al., 2008; Saanouni and Trabelsi, 2016b; Wei, 1996).

When the function $a(x)$ is a smooth on $\bar{\Omega}$ and $f(x, t)=$ $\lambda g(t)$, with the same conditions $(\mathrm{G} 1)-(\mathrm{G} 4)$, the problem 
(1.1) was studied by (Saanouni and Trabelsi, 2016a). The condition $g(0)>0$ was capital in their work.

On the other hand, the problem (1.1) was treated by (Zhou, 2002), when $a$ is a constant function but the asymptotically linear nonlinearity depends on $x$ and $t$. More precisely, the author consider the case when:

(F1) The function $f(x, t)$ is continuous on $\bar{\Omega} \times \mathbb{R}, f(x, t) \equiv$ 0 for $t \leq 0$ and $f(x, t) \geq 0$ for all $t>0$, for all $x \in \bar{\Omega}$

(F2) $\lim _{t \rightarrow 0} \frac{f(x, t)}{t}=p(x)$, with $0 \leq p(x) \in L^{\infty}(\Omega)$ and $\|p(x)\|_{\infty}<\lambda_{1}$, with $\lambda_{1}>0$ is the first eigenvalue of $\left(-\Delta, H_{0}^{1}(\Omega)\right)$

(F3) $\lim _{t \rightarrow+\infty} \frac{f(x, t)}{t}=\ell<\infty$ uniformly in a.e. $x \in \Omega$;

(F4) $\frac{f(x, t)}{t}$ is nondecreasing with respect to $t$ in $(0,+\infty)$, for a.e. $x \in \Omega$.

and he proved also that the bifurcation phenomena occurs.

As a recent work, we can cite that ( $\mathrm{Li}$ and Huang, 2019) a generalized quasilinear Schrödinger equations with asymptotically linear nonlinearities. They supposed that the nonlinearities $f(t)$ depend only on $t$ and they proved that the problem has positive solutions. For the superlinear nonlinearities, the Schrödinger equations was investigated by (Li et al., 2020). Throughout this paper, we assume different type of conditions. The nonlinearity $f(x, t)$ does not have to be positive and it is asymptotically linear ( $\ell$ finite) or super linear at $\pm \infty$. More precisely, we make the following assumptions:

(V1) $f \in C(\bar{\Omega} \times \mathbb{R}) ; f(x, 0)=0$ and $f(x, t) t \geq 0$ for all $(x$, $t) \in \Omega \times \mathbb{R} ;$

(V2) $\lim _{t \rightarrow 0} \frac{f(x, t)}{t}<\lambda_{1}$, uniformly for $x \in \Omega$, where $\lambda_{1}$ is the first eigenvalue of the operator $-\operatorname{div}(a(x) \nabla)$, with Dirichlet boundary condition on $\partial \Omega$

(V3) $\lim _{t \rightarrow 0} \frac{f(x, t)}{t}=\ell$, uniformly for $x \in \Omega$.

(V4) $\lim _{t \rightarrow+\infty} \frac{f(x, t)}{t^{r}-1}=0$, uniformly in $x$ for some $r \in\left(2,2^{*}\right)$, here and hereafter:

$$
2^{*}=\left\{\begin{array}{l}
\frac{2 N}{N-2} \text { if } N>2 \\
+\infty \text { if } N=2 .
\end{array}\right.
$$

Also, the weight function $a(x)$ is nonnegative and for this reason we will use weighted Sobolev spaces.

We remark that a non-trivial solution for the Eq. (1.1) is nonzero a critical point of the following functional:

$$
J(u)=\frac{1}{2} \int_{\Omega} a(x)|\nabla u|^{2} d x-\int_{\Omega} F(x, u) d x
$$

with:

$$
F(x, t)=\int_{0}^{t} f(x, s) d s .
$$

In order to investigate the existence of nonzero critical point of $J$, we will apply Mountain Pass Theorem introduced by (Ambrosetti and Rabinowitz, 1973). The most difficult property that $J$ has to satisfy the compactness condition, which is also called the Palais-Smale condition and often, one requires a technical condition introduced as this introduced in (Ambrosetti and Rabinowitz, 1973; Rabinowitz, 1986) and called Ambrosetti - Rabionowitz condition, that is:

$0<\theta F(x, t) \leq f(x, t) t$, for all $|t| \geq t_{0}$ and $x \in \Omega$,

for some $\theta>2$ and $t_{0}>0$.

Sometimes, other type of condition were made as in the following papers (Costa and Magalhaes, 1994; Costa and Miyagaki, 1995; Jeanjean, 1999; Schechter, 1995; Stuart and Zhou, 1996; 1999). When the nonlinearity is asymptotically linear, we can not suppose the condition $(A R)$ because it gives:

$$
\lim _{t \rightarrow+\infty} \frac{F(x, t)}{t^{2}}=+\infty
$$

and so:

$$
\lim _{t \rightarrow+\infty} \frac{f(x, t)}{t}=+\infty .
$$

In this study, we will not use $(A R)$ or any assumption when we prove the existence of critical point for the functional $J$.

Our results state as follows.

\section{Theorem 1.1}

Assume that (V 1), (V 2) and (V 3) are satisfied and $\ell \in(0,1)$. Then, we have:

(i) If $0<\ell<\lambda_{1}$ and the following condition holds

(V 5) $\frac{f(x, t)}{t}$ is nondecreasing function with respect to $t$ in $(0,+\infty)$ and nonincreasing in $(-\infty, 0)$. Then, there is no solution with one sign for problem (1.1)

(ii) If $\ell>\lambda_{1}$, then the problem (1.1) admit a non-trivial solution 
(iii) If $\ell=\lambda_{1}$ and (V 5) holds, then problem (1.1) admit a positive solution $u \in H_{0}^{1}(\Omega, a)$ (resp. negative solution) if and only if there exists a constant $c_{0}>0$ (resp. $c_{0}<$ $0)$ such that $u=c_{0} \varphi_{1}$ and $f(x, u)=\lambda_{1} u$, with $\varphi_{1}$ is a positive eigenfunction associated to $\lambda_{1}$ (see section 2).

\section{Theorem 1.2}

Assume that (V 1) - (V 5) are satisfied and $\ell=+\infty$. Then the problem (1.1) admit a positive solution.

In the present paper $C$ and $C_{i}$ denotes positive constants, which may change from line to another.

\section{Variational Formulation}

Consider $\Omega \subset \mathbb{R}^{N}, N \geq 2$, a regular bounded open set and throughout this paper, we denote:

$$
\|u\|_{p}=\left(\int_{\Omega}|u|^{p} d x\right)^{\frac{1}{p}}, \text { for } 1 \leq p<\infty \text { and } u \in L^{p}(\Omega) .
$$

Let $a(x) \in L^{1}(\Omega)$ be a nonnegative function and followed by (Calanchi et al., 2017), set:

$$
H_{0}^{1}(\Omega, a)=c l\left\{u \in C_{0}^{\infty}(\Omega) ; \int_{\Omega} a(x)|\nabla u|^{2}<\infty .\right\}
$$

Set:

$$
<u, v>=\int_{\Omega} a(x) \nabla u . \nabla v d x
$$

and the norm:

$$
\|u\|=\left(\int_{\Omega} a(x)|\nabla u|^{2} d x\right)^{\frac{1}{2}} .
$$

Weighted Sobolev spaces have been developed and studied for a long time and we can refer to (Drabek et al., 1997; Kufner, 1985).

For completeness, recall that the space $H_{0}^{1}(\Omega, a)$ is a Hilbert space and the embedding $H_{0}^{1}(\Omega, a) \hookrightarrow H_{0}^{1}(\Omega)$ is continuous and so there exists a constant $C$ such that $\|u\|_{H_{0}^{1}} \leq C\|u\|$ for all $u \in H_{0}^{1}(\Omega, a)$, where $\|u\|_{H_{0}^{1}}$ the standard norm on $H_{0}^{1}(\Omega)$. Also, for $q \in\left[2,2^{*}\right]$ the embedding:

$$
H_{0}^{1}(\Omega, a) \hookrightarrow L^{q}(\Omega)
$$

is continuous but it is compact if $q \in\left[2,2^{*}\right)$.

Let $J: \quad H_{0}^{1}(\Omega, a) \rightarrow \mathbb{R}$ be functional of class $C^{1}$ defined by:

$$
J(u)=\frac{1}{2} \int_{\Omega} a(x)|\nabla u|^{2} d x-\int_{\Omega} F(x, u) d x,
$$

where:

$$
F(x, s)=\int_{0}^{s} f(x, t) d t .
$$

\section{Definition 2.1}

$u \in H_{0}^{1}(\Omega, a)$ is called solution of the Eq. (1.1) if for all $\varphi \in H_{0}^{1}(\Omega, a)$ :

$$
\int_{\Omega} a(x) \nabla u \nabla \varphi d x=\int_{\Omega} f(x, u) \varphi d x,
$$

Hence, a solution of the problem (1.1) can be found as critical point of functional $J$. Before starting the Mountain Pass Theorem, we introduce the following definition.

\section{Definition 2.2}

Let $\mathrm{H}$ be a Banach space and a functional $J \in C^{1}(H, \mathbb{R})$. We say $J$ satisfies the Palais Smale (PS) condition if any sequence $\left\{u_{n}\right\} \subset H$ such that $J\left(u_{n}\right)$ converges in $\mathbb{R}$ and $J^{\prime}\left(u_{n}\right) \rightarrow 0$ in $H^{\prime}$, the dual space of $H$, the sequence $\left\{u_{n}\right\}$ has a convergent subsequence.

\section{Proposition 2.1 (Mountain Pass Theorem (Ambrosetti and Rabinowitz, 1973))}

Let $H$ be a Banach space, $J$ a functional in $C^{1}(H, \mathbb{R})$ satisfies the (PS) condition, $J(0)=0$ and:

(i) There exist $\rho, \alpha>0$ such that $J(u) \geq \alpha$, for all $\mathrm{u}$ in the boundary of $B(0, \rho)$

(ii) There exists $y \in H \backslash B(0, \rho)$ such that $J(y)<0$

Then, the functional $J$ admit a critical point $x \in H$ such that $J(x) \geq \alpha>0$.

At the end of this section, recall that $\varphi_{1}$ denotes a normalised positive eigenfunction associated to the first eigenvalue $\lambda_{1} . \varphi_{1}$ satisfies:

$$
\left\{\begin{aligned}
-\operatorname{div}\left(a(x) \nabla \varphi_{1}\right) & =\lambda_{1} \varphi_{1} \text { in } \Omega \\
\varphi_{1} & =0 \text { on } \partial \Omega \\
\left\|\varphi_{1}\right\|_{2} & =1 .
\end{aligned}\right.
$$

\section{Proof of the Theorem 1.1}

We begin by proving the two geometric properties.

\section{Lemma 3.1}

Assume that (V 1) - (V 3) hold and $\ell \in(0,+\infty)$. Then, we can find two positive numbers $\alpha>0$ and $\rho>0$ satisfying:

$$
J(u)>\alpha, \text { forall } u \in H_{0}^{1}(\Omega, a) ;\|u\|=\rho .
$$




\section{Proof}

Using (V 2), there exist $\varepsilon_{0} \in(0,1)$ and $\delta_{0}$ satisfying:

$$
F(x, t) \leq \frac{1}{2} \lambda_{1}\left(1-\varepsilon_{0}\right) t^{2}
$$

for all $|t| \leq \delta_{0}$.

From (V 3), for $1 \leq q \leq \frac{N+2}{N-2}$, we can have a constant $C>0$ verifying:

$$
F(x, t) \leq C|t|^{q+1}
$$

for all $|t| \geq \delta_{0}$.

So, we have:

$$
F(x, t) \leq \frac{1}{2} \lambda_{1}\left(1-\varepsilon_{0}\right) t^{2}+C|t|^{q+1}, \text { for all t for all } \mathbb{R}
$$

and then:

$$
J(u) \geq \frac{1}{2}\|u\|^{2}-\frac{1}{2} \lambda_{1}\left(1-\varepsilon_{0}\right)\|u\|_{2}^{2}-C\|u\|_{q+1}^{q+1}
$$

Since $\lambda_{1}\|u\|_{2}^{2} \leq\|u\|^{2}$ (by definition of $\lambda_{1}$ ) and by continuous embedding result, we get:

$$
J(u) \geq \frac{1}{2} \varepsilon_{0}\|u\|^{2}-C_{1}\|u\|^{q+1} .
$$

Set $\|u\|=\rho>0$ small enough, we get $J(u) \geq \alpha$, where $\alpha=\frac{1}{2} \varepsilon_{0} \rho^{2}-C_{1} \rho^{q+1}$.

In the next lemma, we prove the second geometry property.

\section{Lemma 3.2}

Suppose that (V 1) and (V 3) hold and $\lambda_{1}<\ell, \ell \in \mathbb{R}$. Then, there exists $w \in H_{0}^{1}(\Omega, a)$, with such that $J(w)<0$ and $\|w\|>\rho$.

\section{Proof}

For $t>0$, consider the function:

$$
\phi(t)=J\left(t \varphi_{1}\right)=\frac{t^{2}}{2}\left\|\varphi_{1}\right\|^{2}-\int_{\Omega} F\left(x, t \varphi_{1}\right) d x .
$$

By (V 1) the function $F(x, t) \geq 0$ and the Fatou's Lemma gives:

$$
\lim _{t \rightarrow+\infty} \frac{1}{t^{2}} \phi(t) \leq \frac{1}{2}\left\|\varphi_{1}\right\|^{2}-\int_{\Omega} \lim _{t \rightarrow \infty} \frac{F\left(x, t \varphi_{1}\right)}{\left(t \varphi_{1}\right)^{2}} \varphi_{1}^{2} d x
$$

It follows from (V 3) that:

$$
\lim _{t \rightarrow+\infty} \frac{1}{t^{2}} \phi(t) \leq \frac{1}{2}\left\|\varphi_{1}\right\|^{2}-\frac{\ell}{2} \int_{\Omega} \varphi_{1}^{2} d x .
$$

So:

$$
\lim _{t \rightarrow+\infty} \frac{1}{t^{2}} \phi(t) \leq \frac{\lambda_{1}}{2}-\frac{\ell}{2}<0 .
$$

We have $\lim _{t \rightarrow+\infty} J\left(t \varphi_{1}\right)=-\infty$ and so there exist $w \in H_{0}^{1}(\Omega, a)$ with $\|w\|>\rho$ and $J(w)<0$.

\section{Proof of the Theorem 1.1}

(i) Suppose that $u$ is a positive solution or negative solution $\in H_{0}^{1}(\Omega, a)$ for (1.1). From the Definition 2.1 and the conditions (V 1) - (V 3) and (V 5), we obtain:

$\int_{\Omega} a(x)|\nabla u|^{2} d x=\int_{\Omega} f(x, u) u d x \leq \int_{\Omega} \ell u^{2} d x$

which gives $\lambda_{1} \leq \ell$. This finish the proof of (i).

(ii) Let $\lambda_{1}<\ell$. If we consider Lemma 3.1 and Lemma 3.2 in mind, we have only to prove the $(P S)$ condition for the functional $J$ given by the formula (2.2). For this, consider $\left\{u_{n}\right\}$ a $(P S)$ sequence of $J$ :

$$
J\left(u_{n}\right)=\frac{1}{2}\left\|u_{n}\right\|^{2}-\int_{\Omega} F\left(x, u_{n}\right) d x,
$$

$J\left(u_{n}\right) \rightarrow d$ as $n \rightarrow+\infty$

for some $d \in \mathbb{R}$ and:

$\left\|J^{\prime}\left(u_{n}\right)\right\|_{*} \rightarrow 0$ as $n \rightarrow+\infty$.

Step 1 (If $\left\{u_{n}\right\}$ is bounded in $H_{0}^{1}(\Omega, a)$, then $\left\{u_{n}\right\}$ is relatively compact)

Suppose that $\left\{u_{n}\right\}$ is bounded in $H_{0}^{1}(\Omega, a)$. By compact embedding result, we get up to subsequence:

$$
\begin{aligned}
& \quad u_{n} \rightarrow u \text {, converges weakly in } H_{0}^{1}(\Omega, a), \\
& u_{n} \rightarrow u \text {, converges strongly in } L^{2}(\Omega) \\
& \text { besides } \\
& u_{n} \rightarrow u \text {, a.e. in } \Omega .
\end{aligned}
$$

where, $\rightarrow$ denotes the weak convergence. From (3.8), we obtain:

$<J^{\prime}\left(u_{n}\right), u_{n}>=\left\|u_{n}\right\|^{2}-\int_{\Omega} f\left(x, u_{n}\right) u_{n} d x \rightarrow 0$ 
and also, for all $\varphi \in H_{0}^{1}(\Omega, a)$ :

$\int_{\Omega} a(x) \nabla u_{n} . \nabla \varphi d x-\int_{\Omega} f\left(x, u_{n}\right) \varphi d x \rightarrow 0$.

So:

$-\operatorname{div}\left(a(x) \nabla u_{n}\right)-f\left(x, u_{n}\right) \rightarrow 0$ in $H_{0}^{-1}(\Omega, a)$

where, $H_{0}^{-1}(\Omega, a)$ indicates the dual space of $H_{0}^{1}(\Omega, a)$. From (V 1) - (V 3), $f\left(x, u_{n}\right) \rightarrow f(x, u)$ in $L^{2}(\Omega)$ and so:

$-\operatorname{div}\left(a(x) \nabla u_{n}\right) \rightarrow f(x, u)$ in $H_{0}^{-1}(\Omega, a)$.

As in (Meyers, 1963), we prove that the operator $L=$ $-\operatorname{div}(a(x) \nabla)$ is an isomorphism between $H_{0}^{1}(\Omega, a)$ and $H_{0}^{-1}(\Omega, a)$ so:

$u_{n} \rightarrow L^{-1}(f(x, u))$ in $H_{0}^{1}(\Omega, a)$.

Step $2\left(\left\{u_{n}\right\}\right.$ is bounded in $H_{0}^{1}(\Omega, a)$

By contradiction. We suppose that the sequence $\left\{u_{n}\right\}$ is unbounded. Up to subsequence, we have:

$$
\left\|u_{n}\right\| \rightarrow+\infty \text { as } n \rightarrow+\infty .
$$

Let:

$$
w_{n}=\frac{u_{n}}{\left\|u_{n}\right\|}, t_{n}=\left\|u_{n}\right\|
$$

The sequence $\left\{w_{n}\right\}$ verifies:

$$
\begin{aligned}
& w_{n} \text { converges weakly to } w \text { in } H_{0}^{1}(\Omega, a) \\
& w_{n} \text { converges strongly to } w \text { in } L^{2}(\Omega)
\end{aligned}
$$

and also:

$$
w_{n} \text { converges to } w \text { a.e in } \Omega
$$

for some $\mathrm{w}$ in the space $H_{0}^{1}(\Omega, a)$. From the condition (V 3), there exists $C_{2}>0$ such that:

$$
\frac{f(x, t)}{t} \leq C_{2}, \text { for all } t \neq 0, \text { and } x \in \Omega
$$

so:

$$
\int_{\Omega} \frac{f(x, t)}{\left\|u_{n}\right\|^{2}} u_{n} d x=\int_{\Omega} \frac{f\left(x, u_{n}\right)}{u_{n}} w_{n}^{2} d x \leq C \int_{\Omega} w_{n}^{2} d x .
$$

From (3.9), the left hand side of (3.15) converge to 1 and then:

$$
w \neq 0
$$

By using (3.10), we get:

$\int_{\Omega} a(x) \nabla w_{n} \cdot \nabla \varphi d x$

$-\int_{\Omega} \frac{f\left(x, u_{n}\right)}{u_{n}} w_{n} \varphi d x \rightarrow 0$ for all $\varphi \in H_{0}^{1}(\Omega, a)$.

By using step 1, we know that:

$\int_{\Omega} a(\omega) \nabla w_{n} . \nabla \varphi d \omega \rightarrow \int_{\Omega} a(\omega) \nabla \omega . \nabla \varphi d \omega$,

for all $\varphi \in H_{0}^{1}(\Omega, a)$. Since $u_{n}(x)=\left\|u_{n}\right\| w_{n}(x)$, we deduce that $\lim _{n \rightarrow+\infty} u_{n}(x)= \pm \infty$, whenever $w(x) \neq 0$. Let:

$$
g_{n}(x)=\left\{\begin{array}{r}
\frac{f\left(x, u_{n}(x)\right)}{u_{n}(x)} \text { if } u_{n}(x) \neq 0 \\
0 \text { if } u_{n}(x)=0
\end{array}\right.
$$

From (3.14), the sequence $\left\{g_{n}\right\}$ is bounded on $\Omega$ and so it is weakly star convergent in $L^{\infty}(\Omega)$, up to subsequence to a function $g$.

By (V 3) and the fact that $u_{n}(x)$ not equal to zero a.e. in $\Omega$, the function $g(x)=\ell$, for a.e $x \in \Omega$. Now, if we consider the second term of (3.16), we have:

$$
\int_{\Omega} \frac{f\left(x, u_{n}\right)}{u_{n}} w_{n} \varphi d x=\int_{\Omega} g_{n}(x) w_{n} \varphi d x
$$

That is:

$\int_{\Omega} \frac{f\left(x, u_{n}\right)}{u_{n}} w_{n} \varphi d x \rightarrow \ell \int_{\Omega} w \varphi d x$.

From (3.16), (3.17) and (3.18), we have:

$\left\{\begin{aligned}-\operatorname{div}(a(x) \nabla w) & =\ell w \text { in } \Omega \\ w & =0 \text { in } \partial \Omega,\end{aligned}\right.$

hence $w=c \varphi_{1}$ and $\ell=\lambda_{1}$. This is impossible since we have supposed that $\lambda_{1}<\ell<\infty$. We deduce $\left\{u_{n}\right\}$ is bounded in $H_{0}^{1}(\Omega, a)$.

Step 3 (Conclusion)

By Proposition 2.1, the Eq. (1.1) has a nontrivial solution. 
(iii) Suppose that $\ell=\lambda_{1}$. Set $\mathrm{u}$ a positive solution for (1.1) or negative solution. If we take $\varphi=\varphi_{1}$ in (2.3), we get:

$\int_{\Omega} a \nabla u . \nabla \varphi_{1}=\int_{\Omega} f(x, u) \varphi_{1}$.

Consider the Eq. (2.4), multiply it by u and integrate, we obtain:

$$
\int_{\Omega} a(x) \nabla u . \nabla \varphi_{1} d x=\ell \int_{\Omega} u \varphi_{1} d x .
$$

From (3.20) and (3.21), we get:

$$
\int_{\Omega}(f(x, u)-\ell u) \varphi_{1} d x=0
$$

From (V 3) and (V 5), the constant $C$ in the inequality (3.14) will be equal to $\ell$ and since $\varphi_{1}>0$, we obtain $f(x$, $u)=\ell u$ a.e. in $\Omega$. This means $f(x, u)=\lambda_{1} u$ and then $u$ is an eigenfunction associated to the simple eigenvalue $\lambda_{1}$, so $u=c \varphi_{1}$ for some constant $c>0$ or $c<0$ according to the sign of $u$.

Conversely, if $\ell=\lambda_{1}, u=c \varphi_{1}$ for some constant $c \neq 0$ and $f(x, u)=\lambda_{1} u$. Then, $u$ is a solution of (1.1).

\section{Proof of the Theorem 1.2}

First, we begin by proving the geometric properties.

\section{Lemma 4.1}

Assume that (V 1), (V 2), (V 4) hold and $\ell=+\infty$. Then, there exist positive numbers $\alpha, \rho$ verifying:

$$
J(u)>\alpha, \forall u \in H_{0}^{1}(\Omega, a) ;\|u\|=\rho .
$$

\section{Proof}

Using (V 4), there exist $C_{2}>0$ and $t_{0} \geq 1$ such that for all $|t| \geq t_{0},|f(x, t)| \leq C_{2}|t|^{r-1}$. As in Lemma 3.1 and by using (V 2), we can find $\varepsilon_{0} \in(0,1)$ such that:

$$
F(x, t) \leq \frac{1}{2} \lambda_{1}\left(1-\varepsilon_{0}\right) t^{2}+C_{2}|t|^{r}, \forall(x, t) \in \Omega \times \mathbb{R} .
$$

Therefore:

$$
J(u) \geq \frac{1}{2}\|u\|^{2}-\frac{1}{2} \lambda_{1}\left(1-\varepsilon_{0}\right)\|u\|_{2}^{2}-C_{2}\|u\|_{r}^{r} .
$$

From the definition of $\lambda_{1}$ and the choice of $r$, we get:

$$
J(u) \geq \frac{1}{2}\|u\|^{2}-\frac{1}{2} \lambda_{1}\left(1-\varepsilon_{0}\right)\|u\|^{2}-C_{2}\|u\|^{r},
$$

that is:

$$
J(u) \geq \frac{1}{2} \varepsilon_{0}\|u\|^{2}-C\|u\|^{r} .
$$

In the inequality (4.2), we choose $\|u\|=\rho>0$ small enough, we get $J(u) \geq \alpha$ for some $\alpha>0$ since $2<r$.

Now, we pass to the second geometric property of the Proposition 2.1.

\section{Lemma 4.2}

Suppose that the function $f$ satisfies (V 1), (V 3), (V 4 ) and (V 5) with $\ell=+\infty$. Then, $I\left(t \varphi_{1}\right) \rightarrow-\infty$ as $t \rightarrow+\infty$, where $\varphi_{1}$ is a normalised positive eigenfunction associated to $\lambda_{1}$.

\section{Proof}

We have:

$$
J\left(t \varphi_{1}\right)=\frac{t^{2}}{2}\left\|\varphi_{1}\right\|^{2}-\int_{\Omega} F\left(x, t \varphi_{1}\right) d x
$$

that is:

$$
J\left(t \varphi_{1}\right)=\frac{t^{2}}{2} \lambda_{1}-\int_{\Omega} F\left(x, t \varphi_{1}\right) d x
$$

From (V 5), we deduce:

$0 \leq 2 F(x, t) \leq t f(x, t)$.

for all $(x, t) \in \Omega \times \mathbb{R}$ and so, for a fixed $x$ the function $\frac{F(x, t)}{t^{2}}$ is nondecreasing. Therefore, it follows from (V 3) that:

$$
\frac{F(x, t)}{t^{2}} \rightarrow+\infty .
$$

So, there exists $b>\lambda_{1}$ and a constant $C_{3}>0$ such that $F(x, t) \geq \frac{b}{2} t^{2}+C_{3}$ for all $t>0$. The equality (4.3) gives

$$
J\left(t \varphi_{1}\right) \leq \frac{t^{2}}{2} \lambda_{1}-\frac{b}{2} t^{2}\left\|\varphi_{1}\right\|_{2}^{2}-C_{3}|\Omega| .
$$

Then:

$$
J\left(t \varphi_{1}\right) \leq t^{2} \frac{\lambda_{1}-b}{2}<0
$$

So, the Lemma 4.2 is proved.

Similar to the proof of the Lemma 2.3 in (Zhou, 2002), we can prove the following result. 


\section{Lemma 4.3}

Assume that the condition (V 5) holds and $\left\|u_{n}\right\|$ is a sequence in $H_{0}^{1}(\Omega, a)$ such that $\left\langle J^{\prime}\left(u_{n}\right), u_{n}\right\rangle \rightarrow 0$. Then, up to a subsequence:

$$
J\left(t u_{n}\right) \leq \frac{1+t^{2}}{2 n}+J\left(u_{n}\right), \forall t>0
$$

\section{Proof of the Theorem 1.2}

Now, we suppose that $f(x, t)$ is superlinear and it is subcritical at $+\infty$. Let us prove that the problem (1.1) has a nontrivial positive solution.

In order to get the result from Proposition 2.1 and with Lemma 4.1 and Lemma 4.2 in mind, we have to prove that the functional $J$ satisfies compactness property.

Let $\left\{u_{n}\right\}$ a $(P S)$ sequence in $H_{0}^{1}(\Omega, a)$ at level $d$, this means that it satisfying the conditions (3.7) and (3.8). Following the same scheme of the proof of Theorem 1.1 result (ii), we have to prove that the sequence $\left\{u_{n}\right\}$ is bounded in $H_{0}^{1}(\Omega, a)$ and the theorem follows. Suppose that $\left\{u_{n}\right\}$ is not bounded and consider:

$$
w_{n}=\frac{u_{n}}{c\left\|u_{n}\right\|} \text { and } s_{n}=c\left\|u_{n}\right\|
$$

where $c>0$ a positive real number.

It is clear that the sequence $\left(w_{n}\right)$ is bounded in $H_{0}^{1}(\Omega, a)$, hence there exists $w \in H_{0}^{1}(\Omega, a)$ such that, we have the following properties:

$$
\begin{aligned}
& w_{n} \rightarrow w \text { in } H_{0}^{1}(\Omega, a) \text { as } n \rightarrow+\infty, \\
& w_{n} \rightarrow w \text { in } L^{2}(\Omega) \text { as } n \rightarrow+\infty,
\end{aligned}
$$

up to subsequence and also:

$$
w_{n}(x) \text { converges to } w(x) \text { for a.e } x \text { in } \Omega \text {. }
$$

Therefore:

$$
w_{n}^{+}(x) \text { converges to } w^{+}(x) \text { a.e. in } \Omega,
$$

and also:

$$
w_{n}^{+} \rightarrow w^{+} \text {in } L^{2}(\Omega),
$$

since for any function $v \in L^{2}(\Omega)$ :

$$
v^{+}=\frac{v+|v|}{2} .
$$

Now, let $\Omega_{+}=\left\{x \in \Omega ; w^{+}(x)>0\right\}$.
From the fact that $u_{n}(x)=c\left\|u_{n}\right\| w_{n}(x)$, we get $u_{n}^{+}(x) \rightarrow$ $+\infty$ a.e. in $\Omega_{+}$. By exploiting (4.7) and $\ell=+\infty$, we obtain that for all $B>0$, there exists $n_{0}$, for all $n \geq n_{0}$ :

$$
\frac{f\left(x, u_{n}^{+}(x)\right)}{u_{n}^{+}(x)}\left(w_{n}^{+}(x)\right)^{2} \geq B\left(w^{+}(x)\right)^{2} .
$$

It follows from (3.9) that:

$$
\begin{aligned}
& \frac{1}{c^{2}}=\lim _{n \rightarrow+\infty}\left\|w_{n}\right\|^{2}=\lim _{n \rightarrow+\infty} \int_{\Omega} \frac{f\left(x, u_{n}\right)}{u_{n}}\left(w_{n}\right)^{2} d x \\
& \geq \lim _{n \rightarrow+\infty} \int_{\Omega_{+}} \frac{f\left(x, u_{n}^{+}\right)}{u_{n}^{+}}\left(w_{n}^{+}\right)^{2} d x \\
& \geq \int_{\Omega_{+}} \lim _{n \rightarrow+\infty} \frac{f\left(x, u_{n}^{+}\right)}{u_{n}^{+}}\left(w_{n}^{+}\right)^{2} d x .
\end{aligned}
$$

We deduce by that:

$$
\frac{1}{c^{2}} \geq B \int_{\Omega_{+}}\left(w^{+}\right)^{2} d x
$$

for all $B>0$ and so mes $\left(\Omega_{+}\right)=\left|\Omega_{+}\right|=0$ where mes is the Lebesgue measure. Then:

$$
w^{+} \equiv 0 \text { in } \Omega \text {. }
$$

Therefore, $\lim _{n \rightarrow+\infty} \int_{\Omega} F\left(x, w_{n}^{+}(x)\right) d x=0$ and we get:

$J\left(w_{n}\right) \rightarrow \frac{1}{2 c^{2}}$ as $n \rightarrow+\infty$.

If we apply Lemma 4.3, we have up to subsequence:

$$
J\left(w_{n}\right)=J\left(t_{n} u_{n}\right) \leq \frac{1}{2 n}\left(1+t_{n}^{2}\right)+J\left(u_{n}\right)
$$

where, $t_{n}=\frac{1}{c\left\|u_{n}\right\|}$. From (4.10), (4.9) and (3.7) we get $\frac{1}{2 c^{2}} \leq d$, this is for any $c>0$.

So, the Theorem 1.2 is proved. 2

The Theorem 1.2 holds also when the function $f(x, t)$ is superlinear and subcritical at $-\infty$ and the proof is the same.

\section{Conclusion}

A weighted elliptic problem with indefinite asymptotically linear nonlinearity is investigated in the present paper. Under suitable conditions, we prove the existence or the nonexistence of nontrivial solutions. Then, we consider the same problem when the nonlinearity is super-linear and in the same time 
subcritical and we prove an existence result. We use variational method in the proof of the existence of such solutions without using the Ambrosetti-Rabionowitz condition (AR) or any other condition of the same type.

\section{Acknowledgement}

The authors wish to thank the reviewers and the handing editor for their suggestions and their important comments.

\section{Authors' Contributions}

All authors equally contributed in this paper.

\section{Availability of Data or Material}

Every data generated during the work is included in the paper.

\section{Ethics}

This article is original and contains unpublished material. The corresponding author confirms that all of the other authors have read and approved the manuscript and no ethical issues involved.

\section{References}

Abid, I., Jleli, M., \& Trabelsi, N. (2008). Weak solutions of quasilinear biharmonic problems with positive, increasing and convex nonlinearities. Analysis and applications, 6(03), 213-227.

Arioli, G., Gazzola, F., Grunau, H. C., \& Mitidieri, E. (2005). A semilinear fourth order elliptic problem with exponential nonlinearity. SIAM journal on mathematical analysis, 36(4), 1226-1258.

Ambrosetti, A., \& Rabinowitz, P. H. (1973). Dual variational methods in critical point theory and applications. Journal of functional Analysis, 14(4), 349-381.

Brezis, H., Cazenave, T., Martel, Y., \& Ramiandrisoa, A. (1996). Blow up for ut-Du=g (u) revisited. Advances in Differential Equations, 1(1), 73-90.

Costa, D. G., \& Magalhaes, C. A. (1994). Variational elliptic problems which are nonquadratic at infinity. Nonlinear Analysis: Theory, Methods \& Applications, 23(11), 1401-1412.

Costa, D. G., \& Miyagaki, O. H. (1995). Nontrivial solutions for perturbations of the p-Laplacian on unbounded domains. Journal of Mathematical Analysis and Applications, 193(3), 737-755.

Calanchi, M., Ruf, B., \& Sani, F. (2017). Elliptic equations in dimension 2 with double exponential nonlinearities. Nonlinear Differential Equations and Applications NoDEA, 24(3), 1-18.
Drabek, P., Kufner, A., \& Nicolosi, F. (1997). Quasilinear Elliptic Equations with Degenerations and Singularities, Walter de Gruyter, Berlin.

Filippakis, M. E., \& Papageorgiou, N. S. (2006). Multiple solutions for nonlinear elliptic problems with a discontinuous nonlinearity. Analysis and Applications, 4(01), 1-18.

Jeanjean, L. (1999). On the existence of bounded PalaisSmale sequences and application to a LandesmanLazer-type problem set on $\mathbb{R N}$. Proceedings of the Royal Society of Edinburgh Section A: Mathematics, 129(4), 787-809.

Kufner, A. (1985). Weighted sobolev spaces. Wiley.

Li, G., \& Huang, Y. (2019). Positive solutions for generalized quasilinear Schrödinger equations with asymptotically linear nonlinearities. Applicable Analysis, 1-16.

Li, G., Huang, Y., \& Liu, Z. (2020). Positive solutions for quasilinear Schrödinger equations with superlinear term. Complex Variables and Elliptic Equations, 65(6), 936-955.

Martel, Y. (1997). Uniqueness of weak extremal solutions for nonlinear elliptic problems. In Houston J. Math.

Meyers, N. G. (1963). An $\$ \mathrm{~L}^{\wedge} \mathrm{p} \$$-estimate for the gradient of solutions of second order elliptic divergence equations. Annali della Scuola Normale Superiore di Pisa-Classe di Scienze, 17(3), 189-206.

Mironescu, P., \& Rădulescu, V. D. (1993). A bifurcation problem associated to a convex, asymptotically linear function. COMPTES RENDUS-ACADEMIE DES SCIENCES PARIS SERIE 1, 316, 667-667.

Mironescu, P., \& Rădulescu, V. D. (1996). The study of a bifurcation problem associated to an asymptotically linear function. Doctorat en Mathématiques Appliquées, 8.

Rabinowitz, P. H. (Ed.). (1986). Minimax methods in critical point theory with applications to differential equations (No. 65). American Mathematical Soc..

Sanchón, M. (2007). Boundedness of the extremal solution of some p-Laplacian problems. Nonlinear Analysis: Theory, Methods \& Applications, 67(1), 281-294.

Schechter, M. (1995). Superlinear elliptic boundary value problems. manuscripta mathematica, 86(1), 253-265.

Saanouni, S., \& Trabelsi, N. (2016a). A bifurcation problem associated to an asymptotically linear function. Acta Mathematica Scientia, 36(6), 1731-1746.

Saanouni, S., \& Trabelsi, N. (2016b). Bifurcation for elliptic forth-order problems with quasilinear source term. Electronic Journal of Differential Equations, 2016(92), 1-16.

Stuart, C. A., \& Zhou, H. S. (1996). A Variational Problem Related to Self-trapping of an Electromagnetic Field. Mathematical methods in the applied sciences, 19(17), 1397-1407. 
Hanadi Zahed and Laila A. Alnaser / Journal of Mathematics and Statistics 2021, Volume 17: 13.21 DOI: 10.3844/jmssp.2021.13.21

Stuart, C. A., \& Zhou, H. S. (1999). Applying the mountain pass theorem to an asymptotically linear elliptic equation on RN. Communications in Partial Differential Equations, 24(9-10), 1731-1758.

Turing, A. (1952). Philosophical the royal biological transqfetions society sciences. Phil. Trans. R. Soc. Lond. B, 237, 37-72.
Wei, J. (1996). Asymptotic behavior of a nonlinear fourth order eigenvalue problem. Communications in Partial Differential Equations, 21(9-10), 1451-1467.

Zhou, H. S. (2002). An application of a mountain pass theorem. Acta Mathematica Sinica, 18(1), 27-36. 